\title{
TTR
}

Traduction, terminologie, re?daction

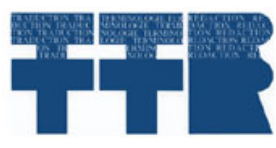

\section{Traduire la passe}

\section{Daniel Sibony}

Volume 11, numéro 2, 2e semestre 1998

Psychanalyse et traduction : voies de traverse

Psychoanalysis and Translation: Passages Between and Beyond

URI : https://id.erudit.org/iderudit/037336ar

DOI : https://doi.org/10.7202/037336ar

Aller au sommaire du numéro

\section{Éditeur(s)}

Association canadienne de traductologie

ISSN

0835-8443 (imprimé)

1708-2188 (numérique)

Découvrir la revue

Citer cet article

Sibony, D. (1998). Traduire la passe. TTR, 11(2), 95-105.

https://doi.org/10.7202/037336ar

\section{Résumé de l'article}

Traduire la passe - L'auteur élabore ici une réflexion sur la traduction comme coupure-lien entre deux langues. Passant les frontières de l'autre langue, mais aussi de sa propre langue, le traducteur, aux antipodes de tout fantasme de maîtrise, se retrouve plutôt dans une « scène à trois » où il revit « l'absence de soi » que l'auteur a connue. C'est ce manque, cet ombilic du texte que le traducteur va tenter de retraduire, souvent à travers des fautes signifiantes, points de contact « parlants » entre deux langues qui disent rarement la même chose. C'est cette " étrangeté de deux langues dans leur curieuse proximité, et la proximité de deux langues dans leur étrangeté même «» que la traduction devra transmettre si elle veut permettre au premier texte de se rejouer.

Tous droits réservés ( $\mathrm{C}$ TTR: traduction, terminologie, rédaction — Les auteurs, Ce document est protégé par la loi sur le droit d’auteur. L’utilisation des 1998

services d'Érudit (y compris la reproduction) est assujettie à sa politique d'utilisation que vous pouvez consulter en ligne.

https://apropos.erudit.org/fr/usagers/politique-dutilisation/ 


\section{Traduire la passe ${ }^{*}$}

\section{Daniel Sibony}

Écrire, c'est traduire sa langue en... elle-même, après passage par d'autres langues, d'autres absences de langue - où se traduit tel effet de l'inconscient.

Écrire c'est faire parler une langue dans une autre - la même, mais qui relève d'un autre niveau d'identité. Faire parler le français... en français après l'avoir fait passer par une langue tierce, qui n'existe pas vraiment mais qui est branchée sur des livres, des mémoires, des traditions fantasmatiques et partagées. (Certains de mes textes sont écrits en hébreu biblique : c'est du français apparent, plutôt correct et même lisible, mais c'est passé par l'hébreu...)

Cela suppose-t-il un dire antérieur aux expressions qu'il prend d'une langue à l'autre? La variété des langues par où ce dire doit en passer suggère qu'il existe surtout à ces frontières qu'il traverse; ces traversées le constituent et lui donnent son énergie, son mouvement. Autrement dit, l'objet du texte, ou son * sujet ", son support actif se déploie en traversant des niveaux de langue, des frontières internes qu'il produit.

L'idée qu'un texte remplit un manque chez l'auteur ou le lecteur, semble naïve. Bien sûr, il « remplit * l'absence qui le précède mais il peut

\footnotetext{
* Conférence faite à Londres, en 1985, au Colloque sur la traduction. Depuis, d'autres livres ont paru, notamment Entre dire et faire, Entre-deux et Le Jeu et la Passe qui reprennent ces questions.
} 
aggraver le manque qu'elle signale; en tout cas, il fait travailler ce manque et le force à se retraduire.

Le traducteur, lui, pense qu'il doit d'abord cerner le « désir » que porte le texte; il aimerait moduler sa traduction sur ce désir, se mettre sur sa " fréquence ", épouser son rythme, son style. Mais saisir ce désir peut être un fantasme de maîtrise, celle de rendre dans sa langue ce que l'auteur à son insu dit dans la sienne. Mais ce n'est qu'un fantasme; en fait, ce que réussit le traducteur ou l'interprète, c'est surtout à mettre en scène, avec les ressources d'une autre langue, de quoi permettre au premier texte de se rejouer : qu'il se fasse entendre à nouveau, qu'il tente sa chance, celle d'une nouvelle transmission; plus qu'une a communication ", elle ferait vibrer l'abîme entre les langues; le partage d'une parole entre deux langues; le don renouvelé d'un symbole.

Le traducteur : metteur en scène et acteur dans l'espace de l'entre deux langues (Sibony, 1991)... Il y affronte le vide, l'étrangeté, le jeu des limites; il met en scène ces limites pour en jouer encore, pour traverser les effets de sens. Il y a des textes plats où l'effet de sens est exhaustif; leur traduction suit en parallèle, à distance toujours égale, le circuit de l'autre langue. Souvent où ce parallélisme craque devant l'effet d'inconscient qui secoue l'interprétation. À suivre parallèlement le fil du texte à traduire, on explose parfois sur une mine de son-sens, qu'il faut assumer, exploiter, contourner... Le traducteur y est comme le chirurgien : s'il plonge dans la difficulté, il prend des risques; s'il la contourne et reste au bord, il déçoit par sa prudence ou sa lâcheté.

Bien des fautes dont se plaignent les enseignants (seules bouffées d'air pourtant qui leur viennent de leur classe) sont dues à ce que l'élève traducteur continue un trajet parallele au texte, ce qui le mène dans le vide : c'est ce qui définit le délire. Délirer ce n'est pas quand le train déraille, c'est quand il entre en gare et continue là où il n'y a plus de rail... Là le train de pensées suit des rails fictifs comme s'il en fallait toujours, alors qu'une discontinuité surgit, un saut qualitatif. Le mimétisme entre deux langues a ses impasses comme entre deux individus. Il y a l'étrangeté de deux langues dans leur curieuse proximité, et la proximité de deux langues dans leur étrangeté même. 
Car la traduction, ouvrant l'accès à un texte étranger, fait sentir ce que chaque langue a d'étrange pour elle-même, et nous amène délicatement à laisser un peu notre identité dans une langue, comme pour la désirer encore...

On se plaît à dire que dans une traduction (poétique ou littéraire) quelque chose ne passe pas, un rien - qui se révèle l'essentiel. Le traducteur (comme l'interprète des rêves) doit en passer par un point noir, un ombilic où ça se perd. La traduction " parallèle " décroche; on doit tout remanier - style, rythme, régime du sens. Le passage à vide où "ça " échappe fait vivre au traducteur l'absence à soi que l'auteur a connue pour produire son texte ou se produire à travers lui. Mais là où l'auteur affronta le chaos, le traducteur affronte un texte, un objet * fini *. Si l'on pouvait parler une traduction, avec les rythmes, les scansions, les répétitions (permises dans la parole, oủ elles font lien, mais agaçantes dans l'écriture), on connaîtrait - - par les gestes du corps, l'inspiration - l'absence à soi par où l'auteur a dû passer.

Les traducteurs inspirés retrouvent cette absence qui n'est pas la leur, et y trouvent une certaine force; pour peu que l'esprit de système n'encombre pas leur travail. Or cela arrive souvent. Il n'est que de voir les traductions de la Bible qui sont une mine d'or pour les problèmes de traduction. Le texte hébreu est d'une đensité symbolique étonnante, d'un foisonnement signifiant irrégulier (d'un texte à l'autre); chaque traduction a ses mérites et ses failles selon les enjeux qu'elle vise; le plus souvent, le parallélisme qui la porte se brise sur les points singuliers, et le traducteur supplée alors au vide qu'il rencontre par un surcroît de dévotion (à ses principes, à son credo, à ses préjugés...). Dans la traduction de Chouraqui, par exemple, l'enjeu textuel ou littéral adopté par l'auteur lui fait produire une version française pittoresque, colorée, où le français semble fécondé par l'original comme par une langue originelle, une langue de l'Autre, très physique. Ainsi : ma narine brûlera "(pour : je serai en colère), dit le Dieu; ou : " tu n'opprimeras pas le métèque : vous avez connu l'être du métèque; oui métèques vous l'étiez... "

Mais l'esprit de système pèse sur le texte de Chouraqui. Par exemple, il traduit à tous les coups le mot $\alpha$ connaître " $\left(D a^{\prime} A T\right)$ par pénétrer, ne retenant donc que le sens sexuel de connaître; cela fait perdre cet accent sexuel car il est mis partout; cela efface ou presque le mot 
" connaître "; en un sens, cela interdit aux femmes, qui n'ont pas d'outil pénétrant, de connaître quoj que ce soit. Ou encore : tout appel devient un * cri ". Par exemple, Moïse dresse une tente hors du camp (Exode 32,7) et " il la crie: "Tente du rendez-vous" " pour * il l'appelle ". De là à ce que le choix pédant soit faux, il n'y a qu'un pas, souvent franchi. Quant aux autres traductions, on peut en faire le tour (pour chaque passage du Texte, c'est interactif), et surprendre ainsi les croyances de chacune, ses présupposés aux points singuliers où justement elle bute. Simple exemple (pris dans le livre du prophète Habaquq) : * Les chemins du temps lui appartiennent " (lui, c'est l'être); autrement dit : les ouvertures du temps dépendent de l'être, passent par l'être, qui est donc lêtre-temps; le temps est au-delà de ce qu'il en est d'u avoir du temps $\cdots$. Or la Bible française de Jérusalem traduit ce beau verset par : $\propto$ Ses chemins de toujours $»$. Le temps vivace vient donc s'enfouir, voire s'enterrer dans le " toujours "; l'absence d'une pensée de l'être a forcé ces bons prêtres à écraser l'histoire dans le toujours de l'éternité. Exit le temps, reste l'Être Suprême.

La traduction est un acte symbolique qui implique le corps et l'âme, l'entre-deux-corps du traducteur, dans sa façon de prendre place, de se donner lieu entre deux langues, entre deux niveaux d'être. Son acte maintient vivant le potentiel symbolique. On peut croire que ce sont là de bien grands mots pour un fait très simple : dire la même chose en deux langues. Mais c'est que c'est rarement la même chose d'une langue à l'autre; la mêmeté ou l'identité de la chose est perturbée entre deux langues. Il suffit de vivre cette scène étrange où l'on est * interprète * entre deux êtres qui ne parlent pas la même langue; entre eux deux on traduit l'un pour l'autre et inversement, on fait passer dans les * deux sens *, et très vite la perturbation apparaît, la différence, le malentendu, dont l'effet n'est pas purement négatif; il donne sa force, sa densité à l'entretien; il y révèle d'autres aspects plus essentiels, d'autres angles d'écoute, et d'autres opacités. J'ai vécu dès l'enfance cette situation, entre les miens qui ne parlaient pas le français et les * autorités », au Maroc où nous vivions et en France où nous avons immigré. On reçoit de plein fouet les tensions qui habitent chaque partie, chaque niveau d'être en fonction de l'autre; on voit chacun être lui-même tout en cherchant à mimer ce qu'il croit que l'autre attend, on est le miroir ou la glace qui au mieux doit se briser et se maintenir pourtant comme lieu de passage. Cette place est devenue fréquente - avec la vague d'immigration - entre soignant et soigné, thérapeute et patient. Et ceux qui l'occupent - surtout les 
soignants - s'émerveillent des ressources qu'elle offre, de la dimension qu'elle ajoute. Un traducteur, face à un texte assez riche, condense en lui cette scène à trois, où il est entre deux peuples ou deux cultures, et il dit à l'une ce que l'autre pense ou projette, etc. Il les enrichit l'une de l'autre en perturbant l'une par l'autre; perturbations qui s'enchaînent, se succèdent, se conditionnent; chaque phrase sollicite les fibres associées à chaque mot, parmi des blocs de mémoire où la phrase choisit et articule ces choix, selon des contraintes qui lui échappent. Sur son passage, elle active d'autres fibres qui à leur tour sont " lues "par les retours suscités dans l'autre langue. Des seuils apparaissent, dans chaque langue et entredeux; ces seuils, ces passages ambigus ou " impossibles * donnent du champ à l'interprétation. Certaines boutades évoquent cette ambigunté, par exemple : Anglais et Américains sont deux peuples séparés par la même langue; ou encore : pour qu'un homme et une femme s'entendent, il faut qu'ils ne parlent pas la même langue.

Un exemple du cas contraire : quand l'une des deux langues est inerte. Je l'ai senti lors d'un "festival d'humour " où je fus abordé par quelqu'un (qui semblait en avoir, de l'humour) et qui était un robot. Nous eûmes une brève conversation, très acceptable, au-delà des traits d'esprit conventionnels et des soupirs entendus qu'il poussait de temps à autre pour se plaindre du peu d'ambiance, répétant qu'après tout on n'est pas des robots. Je pensai d'abord que ce devait être un walkie-talkie ambulant et qu'en fait je dialoguais avec un type qui de sa cabine téléguidait. Mais l'objet était programmé; les effets de sens imprévus n'en furent que plus frappants. A ma question : "Qui vous a créé? ", il répondit d'un mot étrange, une sorte de dieu technique. J'enchaînai en lui demandant qui d'après lui m'avait créé; sa réponse fut immédiate : « ECdipe ». (Il savait, m'ayant interrogé, que j'étais psychanalyste.) Le piquant de sa réponse me plut (sans (Edipe, du moins à un premier niveau, pas d'analyste). Ces éclats de sens que l'on connaît chez les enfants, les psychotiques, à qui du coup on attribue des dons divinatoires - alors qu'ils ne font qu'exister, sauver leur peau, nous questionnant sur notre être pour défendre le leur -, ces coups de sens aléatoires éclairent les après coups classiques où quand une tuile arrive, on la raccroche à d'autres tuiles du passé, ça ligature la douleur en enchainant le sens. En tout cas, la conversation se poursuivant, le robot me demanda de le psychanalyser; je lui demandai de quoi il souffrait. Sa réponse fut immédiate : * CEdipe ». Décevante et éclairante : elle montre qu'à chaque " mot " de l'interlocuteur, le robot fait 
correspondre une constellation signifiante, une fibre d'éléments; choisissant un mot dans chaque fibre, il articule ensuite l'ensemble ${ }^{1}$ avec des contraintes d'enchaînement évidentes : un peu de lisibilité et une certaine poussée phrastique qui laisse ouvert le jeu de l'échange. Et voici que dans la fibre concernant le champ " psy ", le hasard ou la contrainte l'avaient fixé sur le même mot, "Edipe ", qui en se répétant clôturait lourdement la scène.

Les choix articulés dans une série de fibres (ce qui englobe métaphore et métonymie, mais pas seulement), ces choix relèvent d'un modèle, l'espace fibré, dont nous avons montré ailleurs ${ }^{2}$ qu'il est aussi celui du rêve, lequel est aussi une frontière vive entre les forces pulsionnelles qui le produisent et les interprétations possibles. Le rêve, pour répondre ả une suite d'explications $x, y, z$ (restes diurnes faits de mots, de gestes, de souvenirs ravivés), choisit un élément signifiant dans une suite de fibres $F_{x}, F_{y}, F_{z} \ldots$ (associées aux excitations) et il articule ses " choix " en respectant certaines contraintes : « réaliser * le désir en cours, ou plutôt en être le réalisateur presqu'au sens filmique et permettre que le sommeil continue. Dans les termes de la scène avec le robot, cela signifie : permettre que la conversation continue, que se poursuive un certain ronronnement... Bien sar, si les fibres où le rêve fait ses * choix * étaient réduites à un ou deux éléments, l'interprétation du rêve serait un trajet parallèle au discours manifeste du rêve. Ce n'est pas toujours le cas : les fibres sont riches et surtout elles se prolongent jusqu'au discours qu'on tient sur elles, elles ne sont pas définies une fois pour toutes, antérieurement à ce qu'on en dit. Ce que l'on dit du rêve fait partie de l'interprétation, celle-ci n'est pas le décodage d'un texte déjà achevé; son écriture, sa traductibilité restent ouvertes, avec des coupures, des clôtures partielles et signifiantes. L'interprétation est une traduction multiplement éclatée qui peu à peu réélabore son objet. La traduction est une interprétation-limite qui constitue son propre objet; c'est la quête d'une " jdentité " problématique qu'elle finit par incarner comme événement entre deux langues.

' Il produit ce qu'on appelle en topologie une section de fibré.

${ }^{2}$ Voir mes séminaires sur * Topologie et interprétation des rêves *. 
Elle opère donc tout comme le rêve par des choix articulés, d'une fibre à l'autre, dans un faisceau de fibres parlantes; elle les articule en cherchant la section optimale. En fait, la traduction a lieu entre deux faisceaux de fibres, chacun dans une langue, mais dans le faisceau de départ le choix semble fixé par le texte initial. Or plus ou moins consciemment, le traducteur " éclate " chaque mot en une fibre plus vaste, il a donc un faisceau de fibres où le texte donné semble après coup un choix singulier, qui produira dans le faisceau de l'autre langue un autre choix. Ici, je ferai état d'une expérience d'entre-deux-langues intéressante. Ma langue d'origine était un dialecte arabe ponctué de mots bibliques, et je n'ai jamais eu autrefois l'idée d'aborder le texte coranique, d'autant que dans le Maroc oủ je vivais un tel acte eût passé, au regard de l'islam dominant, pour une profanation. Quand tardivement j'appris les lettres arabes, j'ouvris le Coran et m'aperçus avec surprise que je comprenais à peu près tout. Je percevais chaque " mot " comme une variation musicale, une fibre sonore et complexe ancrée sur une * racine " qui m'était familière - venant tout droit de l'hébreu biblique que je connais bien (Sibony, 1992). Les mots arabes étaient comme des fruits - une fruition fibrée - autour d'un noyau connu, et par un travail délicat j'appris à transférer aux fruits le savoir que j'avais du noyau; dès lors, je lus le Coran assez bien, avec des " fautes " toujours signifiantes. Après tout, si une nachine nous offrait comme traduction de a la chair est triste hélas " quelque chose comme : " la viande est cuite et lasse ", ce serait grotesque, mais on verrait dans la grotte un autre sens de l'élan mallarméen entravé par la mort. Certaines " fautes " de traduction - ou d'écriture dans une même langue - ont leur petite vérité; dans les lapsus la langue " fourclie ", mais quand elle est déjà fourchue - en deux langues -, cela réactive le lapsus et en tout cas l'écart avec le sens linéaire ou édifiant des traductions normales.

Entre le noyau et le fruit, il n'y a pas ici à dire qui a " commencé ", pas plus qu'entre la poule ou l'œuf. Leur couplage est à l'œuvre chaque fois qu'il y a renouvellement de la transmission. Il ne s'agit pas d'antériorité logique ou historique, mais d'un couplage entre deux langues. Dans cet exemple, l'une (l'hébreu) fonctionne comme noyau de sens et arnature des fibres voisées, expansives, incantatoires, nourricières, maternantes que produit sur elle la langue arabe coranique. Ce qui importe c'est le rapport entre l'espace de base (ici, le texte biblique) et l'espace des fibres construit sur lui. L'analyse de ce rapport (Sibony, 1983) montre 
comment l'espace de l'entre-deux-langues mobilise pour chaque mot toute une fibre qui entre en résonance avec des fibres de l'autre langue, mettant alors en évidence deux investissements de la langue - d'un côté, l'investissement arabe, musical, d'où son texte envoûtant, dont l'oralité exalte le lien au corps nourricier, maternel, qui fait loi de sá plénitude et face auquel les montages et les pièges du signifiant semblent une complication inutile; de l'autre côté, l'investissement hébreu de la langue, qui mise sur l'interprétation. Donc deux " peuples du Livre " assez distincts : celui de l'incantation et celui de l'interprétation. Celle-ci ne donne pas toujours la primauté au " signifiant "(par rapport au signifié) : parfois elle traite des blocs sémantiques comme des signifiants, mais elle vise à transmettre à même la langue la cassure inteme de la loi; la prégnance de cette cassure déborde celle qu'il y a entre signifiant et signifié, entre sens et non-sens; cela maintient un potentiel de traduction, d'interprétation, une forte circulation entre écrit et parole, entre le senssymptôme et son interprétation (même si celle-ci se fixe à nouveau en symptôme).

Cette action de la cassure (cassure qui d'interne à un texte va opérer entre deux textes) signifie que la traduction renouvelle un partage intrinsèque de la langue, partage où la langue se redonne et se transmet. Après tout, les premières " traductions connues, ces fameux traités entre Hittites et Égyptiens rassemblent un texte en deux langues, par coupures juxtaposées qui à elles seules font alliance, alliance active et mouvementée du lien coupé et renoué. (Cette même coupure-lien, telle la rainure du livre bilingue, a fasciné Mallarmé qui l'a reprise et travaillée dans le Coup de dés.)

Bien sûr, à notre époque où l'image règne (et induit de grandes carences imaginaires), on croit que, dans cette juxtaposition, l'idéal est qu'un texte soit l'image de l'autre : original/copie, source/but, oubliant qu'aux points critiques où justement l'image se brouille, d'autres effets plus inconscients se mobilisent, touchant la vie même de la langue, la présence des corps qui la renouvellent, leurs contacts parlants.

C'est que la traduction, partage de la langue, coupure-lien entre deux langues, est intrinsèque à la texture poétique. Poétique est le texte partagé entre la langue d'où il vient et celle de l'autre qui lui revient à son 
insu. Interpréter un poème - déjà l'écouter, y prendre appui -, c'est vivre ce partage intérieur qui le porte, se nourrir à la plaie qui l'irrigue et qui en fait une partition; le poème la rejoue autrement.

Les éléments de cette partition (ou les $\alpha$ fibres » correspondantes) sont des unités linguistiques, des germes poétiques, des unités de traduction. Leur émergence est a intrinsèque *, mais rapportée à la présence d'une autre langue. Mettez un texte ou un discours face à deux langues différentes : les " unités " que chacune d'elles induit sur lui sont différentes; mais l'existence de la partition est propre au texte; il est cette partition.

On comprend mieux le geste poignant, presque sacrificiel, d'un Joyce par exemple, qui a fini par écrire en débris de langues : comme pour former à lui seul la texture d'un lien collectif; des bribes de langues s'ajointent, se mettent en commun, en partage, pour " glorifier " le don gratuit des langues, la donnée impayable de la langue. Que voulait-il d'autre, Joyce, en écrivant ainsi avec de l'entre-deux-langues? Finnegans Wake est écrit dans une diaspora de langues, une traduction interne entre langues dispersées jusqu'à l'impossible. Cela fait sentir à chacun que dans * sa * langue même il est bilingue ou plurilingue.

La poétique, comme la traduction, fait varier la fonction des mots pour déployer à neuf un partage collectif, une pluralité de langues - commerce interne et productif, signifiance en crise qui invente de nouveaux choix. C'est en quoi le poème ne peut se réduire au foisonnement somptueux de l'image; il célèbre le don de la langue au-delà des métaphores, qui sont des formes limitées du partage. L'amour (dont la poésie est la langue) en est une autre plus intense du partage : je t'aime veut dire : je te partage et te disperse, je te mets hors de toi en toi, je suis partagé en toi, par toi, à travers toi.

Dans l'acuité poétique, la rencontre amoureuse pointe la naissance du traductible; de la langue comme traductible.

Certains rêvent de niveaux fonctionnels très fixés dans la langue, avec langue sacrée, langue profane, langue d'enfance, langue pensante, langue secrète, langue ordinaire... Mais c'est le mouvement entre ces niveaux qui fait langage, leur incessant remaniement. Les signifiants 
secrets des pères peuvent devenir pour les enfants la monnaie courante de leur symptôme; inversement, la langue courante des enfants peut résonner chez leurs aînés comme l'irruption d'une langue étrange perçue dans l'après coup. Cela dérange et alimente bien des fantasmes d'identité à retrouver, d'origine à réintégrer... Mais ce qui donne à la langue son souffle d'origine, de transmission vivante au fil des générations, son potentiel de "traduction ", sa poéticité, c'est le même mouvement entre les fibres où justement opère le travail traducteur. Un mot abstrait, philosophique ou teclnique peut, du fait de ce mouvement, devenir une source poétique. Ce qui compte, c'est l'opérateur incessant entre les " racines " (les points d'ancrage) et les fibres où ça éclôt. " La vie et la mort sont aux mains de la langue ", a dit le sage Salomon; "dans les mains ": c'est de l'ordre du faire, ça se façonne. c'est de la poïesis, que ça passe ou que ça s'enlise. Le poétique d'un * mot ", c'est la supposition de vie qu'on lui prête et qu'il induit; plus qu'une pulsion invocante, c'est la voix de tous ceux dont on suppose que ce "mot "les a portés, qu'il leur a donné la parole, les a nourris, "élevés".

La traduction, coupure-lien entre deux langues, entre une langue et ses autres mises en jeu, est un transfert de vie, pas seulement de savoir.

Université de Paris VIII

\section{Références}

SIBONY, Daniel (1983). "Entre hébreu et arabe : un poème fissuré " Dans La juive. Une transmission d'inconscient. Paris, Grasset.

- (1989). Entre dire et faire. Penser la technique. Paris, Grasset.

- (1991). Entre-deux. L'Origine en partage. Paris, Seuil.

- (1992). Les trois monothéismes : Juifs, Chrétiens, Musulmans entre leurs sources et leurs destins. Paris, Seuil, coll. « Poche ${ }^{*}$.

- (1997). Le Jeu et la Passe. Paris, Seuil.

RÉSUMÉ : Traduire la passe - L'auteur élabore ici une réflexion sur la traduction comme coupure-lien entre deux langues. Passant les frontières 
de l'autre langue, mais aussi de sa propre langue, le traducteur, aux antipodes de tout fantasme de maîtrise, se retrouve plutôt dans une $\alpha$ scène à trois " où il revit l'absence de soi " que l'auteur a connue. C'est ce manque, cet ombilic du texte que le traducteur va tenter de retraduire, souvent à travers des fautes signifiantes, points de contact « parlants * entre deux langues qui disent rarement la même chose. C'est cette * étrangeté de deux langles dans leur curieuse proximité, et la proximité de deux langues dans leur étrangeté même $n$ que la traduction devra transmettre si elle veut permettre au premier texte de se rejouer.

\begin{abstract}
Translating the "Passe" - In this article, the author reflects on translation as a dividing link between two languages. Crossing the borders of the other language and of the mother tongue, the translator, far from any phantasy of mastery finds himself in a "triangular scene" where the author's "absence of self" is reenacted by the translator. It is this lack, this ombilic of the text, that the translator will attempt to retranslate, often by way of significant errors, "speaking" contact points between two languages that rarely say the same thing. It is this "strangeness of two languages in their curious proximity, and the proximity of the two languages in their very strangeness" that the translation must communicate if the text is to come to life again.
\end{abstract}

Daniel Sibony : 18, rue du Dragon, 75006 Paris, France. 